\section{Case Reports in Ophthalmology}

\title{
Successful Treatment of Lasiodiplodia theobromae Keratitis - Assessing the Role of Voriconazole
}

\author{
Stephen Tak-lun Li Evan Po-fat Yiu Angela Hiu-yan Wong \\ John Chun-Ting Yeung Lester Wang-hon Yu \\ Tuen Mun Eye Centre, Tuen Mun, Hong Kong SAR
}

\author{
Keywords \\ Lasiodiplodia theobromae $\cdot$ Keratitis $\cdot$ Voriconazole $\cdot$ Fungus
}

\begin{abstract}
Purpose: The aim of this paper is to report the successful management of the first case of Lasiodiplodia theobromae keratitis in Hong Kong. Methods: We conducted a case report. Results: A 43-year-old Chinese male with a history of diabetes developed left eye keratitis after a trauma during tree felling. Fungal keratitis was diagnosed using a confocal microscope on day 1 , and L. theobromae was confirmed from the culture. He was given oral voriconazole, topical natamycin, and topical and intracameral amphotericin B. The patient's condition improved after the initial treatment. However, there was a slow progression to descemetocele formation and impending perforation due to corneal melting. Penetrating keratoplasty was performed at 8 weeks after presentation. Final visual recovery was good with no recurrence of infection. The cornea remained clear. Conclusions: We report the first case of $L$. theobromae keratitis in Hong Kong, and it is the only case so far that involved the use of oral voriconazole in the combination therapy. Early recognition with the aid of confocal microscopy allowed the early start of treatment. The use of newer antifungal voriconazole topically and orally combined with topical amphotericin B appeared to be useful in the eradi-
\end{abstract}




\section{Case Reports in Ophthalmology}

Li et al.: Successful Treatment of Lasiodiplodia theobromae Keratitis - Assessing the Role of Voriconazole

cation of the fungus and prevention of recurrence. Intracameral antifungals might have improved the clinical management.

(C) 2016 The Author(s)

Published by S. Karger AG, Basel

\section{Background}

Lasiodiplodia theobromae is a dematiaceous fungus belonging to the subphylum Pezizomycotina of Ascomycota. It is a common plant pathogen causing rot in fruits and plants, mainly in tropical regions [1]. Human infection is extremely rare. Only around 50 cases of $L$. theobromae keratitis have been reported [2].The keratitis can be serious and rapidly progressive with a high chance of corneal perforation, leading to endophthalmitis and panophthalmitis. Management is often difficult as the keratitis shows variable response to antifungals, and there are limited publications for reference. We are reporting the management of the first case in Hong Kong.

\section{Case Report}

A 43-year-old Chinese male construction-site worker presented with acute-onset left eye pain and blurring of vision, which had lasted for 4 days. He had been cutting trees and then rubbed his eye 1 week before the pain started. The patient had a history of diabetes mellitus. Initially, he consulted a private ophthalmologist and was treated with topical dexamethasone and neomycin for 4 days. Unfortunately, his left eye condition worsened. At presentation, his visual acuity was hand movement. Slit-lamp examination showed a left eye stromal infiltrate of $3 \mathrm{~mm}$ in diameter with feathery edges and an overlying epithelial defect of the same size (fig. 1). Confocal microscopy showed fungal hyphae in the superficial and mid-stroma (fig. 2).

The patient was treated empirically as fungal keratitis with topical 5\% natamycin, moxifloxacin eye drops hourly, and oral itraconazole $200 \mathrm{mg}$ daily.

Gram staining showed fungal elements. Culture using Sabouraud glucose neopeptone agar yielded $L$. theobromae 10 days after initial presentation. The clinical condition was static without obvious improvement. His treatment was switched to oral voriconazole $200 \mathrm{mg}$ four times per day. Topical amphotericin B $0.15 \%$ hourly was added, and one injection of intracameral amphotericin B $(7.5 \mu \mathrm{g} / 0.1 \mathrm{ml})$ was given. The keratitis showed improvement with decreased infiltrate, and the lesion size reduced to $2 \mathrm{~mm}$. The topical amphotericin $\mathrm{B}$ and natamycin were tapered to every $2 \mathrm{~h}$ at 5 weeks after presentation.

However, at 6 weeks after presentation, clinical deterioration was noted with new-onset trace hypopyon. Topical amphotericin B was increased to $0.25 \%$ hourly, and oral voriconazole was continued. A second intracameral amphotericin B injection $(7.5 \mu \mathrm{g} / 0.1 \mathrm{ml})$ was given.

At 7 weeks after initial presentation, the hypopyon subsided. The infiltrate reduced in size and became less intense. However, there was a small descemetocele at the periphery of the lesion, and a shallow anterior chamber was noted. Penetrating keratoplasty was performed, and intracameral voriconazole $(50 \mu \mathrm{g} / 0.1 \mathrm{ml})$ was given at the end of the operation.

Postoperatively, topical steroid was withheld for 2 weeks, and topical $1 \%$ voriconazole was given hourly with slow tapering over a span of 3 months. Voriconazole $200 \mathrm{mg}$ twice daily was also given orally after the operation for 3 weeks. At 15 months of follow-up, visual acuity was 6/30, and the cornea graft was clear with a posterior subcapsular cataract (fig. 3). 
The histology report showed that no fungal elements were found on the corneal button using Grocott stain and Masson's trichome stain.

\section{Discussion}

L. theobromae keratitis is rare. Only a few case reports have given details of treatments and outcomes. Clinical response from medical therapy alone has been reported in 3 cases with the use of topical natamycin alone [3], and in 1 case combined with topical amphotericin B [4]. Treatment outcomes using azoles were poor, with disease progression to endophthalmitis and panophthalmitis [5-8]. Surgical treatment by keratoplasty was often required $[2,8]$. However, all cases were complicated by recurrence of infection or graft failure. Except in some mild cases $[3,4]$, the final visual outcomes were generally poor.

The poor outcomes could be explained by the virulence of the fungus. L. theobromae replicates rapidly and is capable of producing a $90-\mathrm{mm}$ colony in 1 week in vitro [6]. It has been postulated that the fungus can secrete collagenases, which lead to corneal melting. In addition, ocular isolates of $L$. theobromae have been shown to be resistant to many antifungals, such as miconazole, ketoconazole, itraconazole, econazole, and natamycin in vitro. In vitro activity was only shown in voriconazole $(1 \mu \mathrm{g} / \mathrm{ml})$ and amphotericin $B(4 \mu \mathrm{g} / \mathrm{ml})$ [2].

Voriconazole is a new-generation triazole with fungicidal in vitro activity against $L$. theobromae. It has good ocular penetration and a low minimum inhibitory concentration $(1 \mu \mathrm{g} /$ $\mathrm{ml}$ ). The therapeutic aqueous drug level can be achieved at a dosage of $200 \mathrm{mg}$ twice daily orally or topical 1\% hourly. [9] Use of voriconazole has only been reported in 3 cases. All 3 cases showed a poor response to topical voriconazole, with 2 cases requiring keratoplasty and 1 case requiring repeated injections of intracameral voriconazole [2, 8].

The case reported herein is the first case involving the use of oral voriconazole early in the management. Although keratoplasty was required, the combined use of topical amphotericin B, natamycin, and oral voriconazole was able to eradicate the fungus, which was proved by the absence of fungal elements in the histopathology of the corneal button. The sterilization of keratitis before cornea transplant decreases the chance of recurrent infection and graft failure. In addition, steroid eye drops were withheld for 2 weeks after the operation, and the use of postoperative topical and systemic voriconazole also helped to reduce the chance of recurrent infection.

Intracameral injection of amphotericin B was used instead of voriconazole because of the author's prior favorable experience with the use of this drug. In addition, the preparation of intracameral voriconzole was initially unavailable from our pharmacy.

Concerning the safety of intracameral injection of amphotericin B, published case series involving the use of the drug at concentrations of 5-10 $\mu \mathrm{g}$ in $0.1 \mathrm{ml}$ do not report any major complications such as corneal toxicity $[10,11]$. A recent randomized controlled trial assessing the efficacy and safety of intracameral amphotericin B showed an increased incidence of cataract as the major complication and it was statistically insignificant [12].

Intrastromal injection of antifungals was not considered in this case because of the presence of a small area of corneal thinning, which would carry a risk of corneal perforation.

This case highlights the importance of the use of confocal microscopy. Not only did it aid early diagnosis before culture results, it also facilitated assessment of the depth of involvement and monitoring of the progress of the disease. In addition, confocal microscopy could also aid the assessment of fungal clearance to determine the best timing to start steroid treatment after keratoplasty. 
Li et al.: Successful Treatment of Lasiodiplodia theobromae Keratitis - Assessing the Role of Voriconazole

\section{Conclusions}

L. theobromae keratitis is a rare infection but can cause serious keratitis leading to blindness. We reported the first case in Hong Kong, and it is the only case involving the use of oral voriconazole in the combination therapy. Further research and case reports are awaited to develop a proper treatment protocol.

\section{Statement of Ethics}

Since this is a retrospective case report, which does not include any experimental treatments or interventions, the need for approval has been waived from the Ethics Committee of the Hospital Authority of Hong Kong. Consent to participate and publish the study has been obtained from the participant.

\section{Disclosure Statement}

The authors declare that they have no competing interests to disclose.

\section{References}

1 Thomas PA: Current perspectives on ophthalmic mycoses. Clin Microbiol Rev 2003;16:730-797.

2 Saha S, Sengupta J, Banerjee D, et al: Lasiodiplodia theobromae keratitis: a case report and review of literature. Mycopathologia 2012;174:335-339.

-3 Rebell G, Forster RK: Lasiodiplodia theobromae as a cause of keratomycoses. Sabouraudia 1976;14:155170.

4 Samudio M, Lapsing F, Farina N, et al: Keratitis by Lasiodiplodia theobromae: a case report and literature review (in Spanish). Rev Chilena Infectol 2014;31:750-754.

5 Donnio A, Desbios N, Boiron P, et al: Mycotic keratitis and endophthalmitis caused by unusual fungi: Lasiodiplodia theobromae (in French). J Fr Ophtalmol 2006;29:e4.

-6 Thomas PA: Current perspectives on ophthalmic mycoses. Clin Microbiol Rev 2003;16:730-797.

-7 Borderie VM, Bourcier TM, Poirot JL, et al: Endophthalmitis after Lasiodiplodia theobromae corneal abscess. Graefes Arch Clin Exp Ophthalmol 1997;235:259-261.

-8 Lekhanont K, Nonpassopon M, Nimvorapun N: Treatment with intrastromal and intracameral voriconazole in 2 eyes with Lasiodiplodia theobromae keratitis: case reports. Medicine (Baltimore) 2015;94:e541.

-9 Hariprasad SM, Mieler WF, Lin TK, et al: Voriconazole in the treatment of fungal eye infections: a review of current literature. Br J Ophthalmol 2008;92:871-878.

10 Yoon KC, Jeong IY, Im SK, et al: Therapeutic effect of intracameral amphotericin B injection in the treatment of fungal keratitis. Cornea 2007;26:814-818.

11 Kaushik S, Ram J, Brar GS, et al: Intracameral amphotericin B: initial experience in severe keratomycosis. Cornea 2001;20:715-719.

12 Sharma N, Sankaran P, Agarwal T, et al: Evaluation of intracameral amphotericin B in the management of fungal keratitis: randomized controlled trial. Ocul Immunol Inflamm 2015;23:1-5. 
Case Reports in
Ophthalmology

Case Rep Ophthalmol 2016;7:179-185

DOI: 10.1159/00044936

c 2016 The Author(s). Published by S. Karger AG, Basel www.karger.com/cop

Li et al.: Successful Treatment of Lasiodiplodia theobromae Keratitis - Assessing the Role of Voriconazole

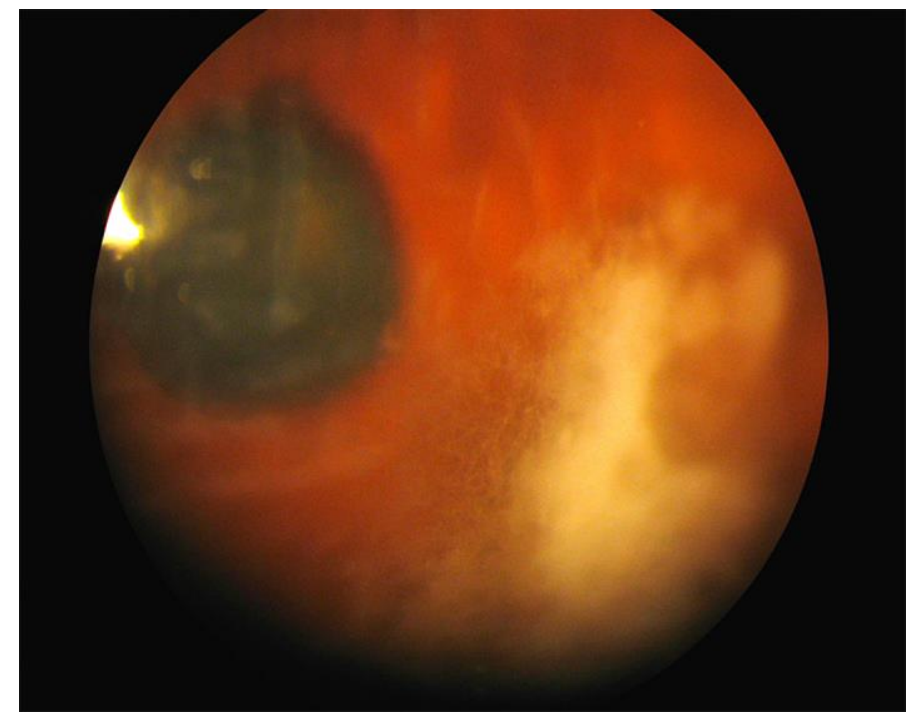

Fig. 1. Corneal infiltrate of $3 \mathrm{~mm}$ in superficial to mid-stroma with visible fungal hyphae at the periphery on slit-lamp examination. 
Case Reports in
Ophthalmology

Case Rep Ophthalmol 2016;7:179-185

\begin{tabular}{l|l}
\hline DOI: 10.1159/000449369 & ( 2016 The Author(s). Published by S. Karger AG, Basel \\
\hline
\end{tabular} www.karger.com/cop

Li et al.: Successful Treatment of Lasiodiplodia theobromae Keratitis - Assessing the Role of Voriconazole

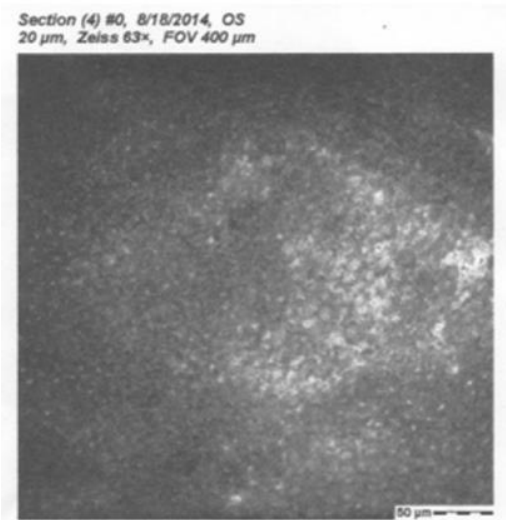

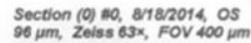

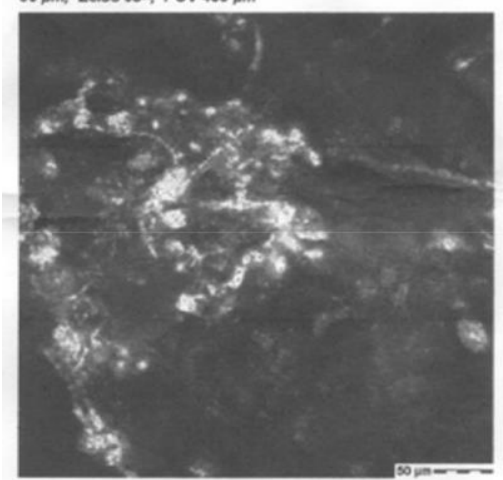

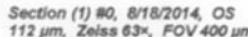

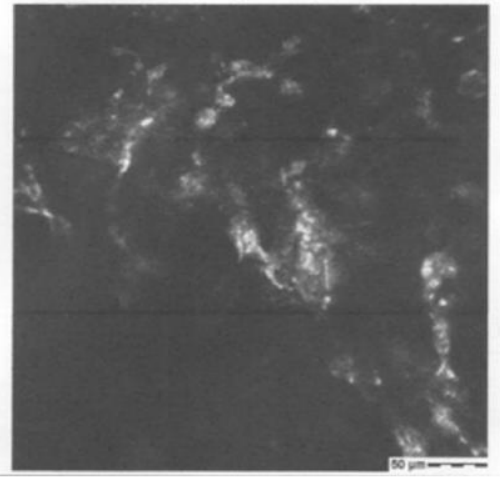

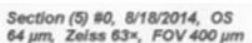

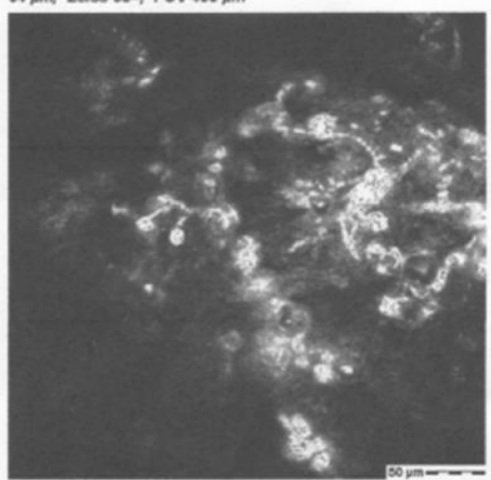

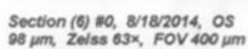

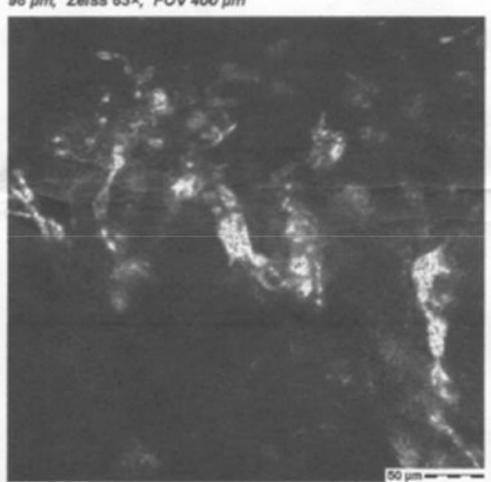

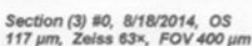

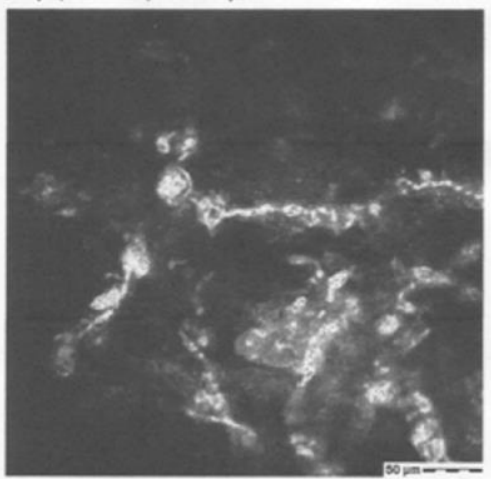

Fig. 2. Specular microscopy showing fungal hyphae in superficial to mid-stroma (64-117 $\mu \mathrm{m}$ in depth). 
Case Reports in
Ophthalmology Case Rep Ophthalmol 2016;7:179-185 DOI: $10.1159 / 000449369$

(C) 2016 The Author(s). Published by S. Karger AG, Basel www.karger.com/cop

Li et al.: Successful Treatment of Lasiodiplodia theobromae Keratitis - Assessing the Role of Voriconazole

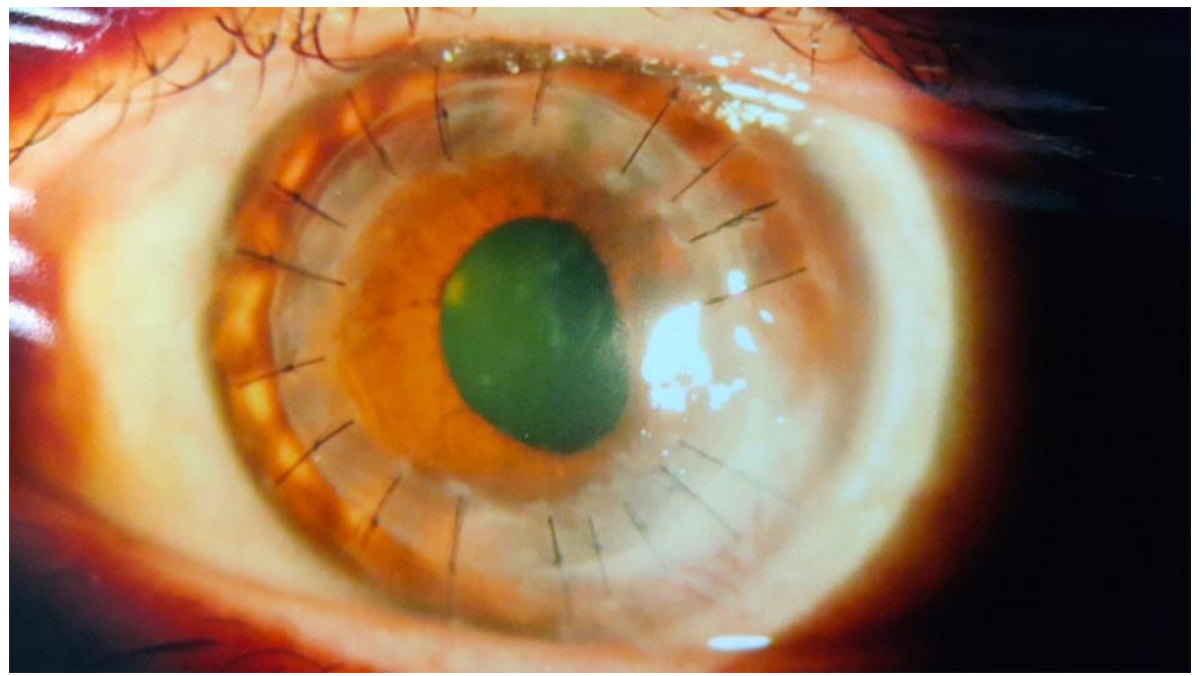

Fig. 3. Clear cornea maintained at 4 weeks after keratoplasty with no signs of recurrent infection. 